\title{
The Reimann Hypothesis Clay Institute Millenium Problem Solution
}

\author{
PAUL T E CUSACK* \\ Independent Researcher, BSc E, DULE, 1641 Sandy Point Rd, Saint John, NB, Canada E2K 5E8, Canada \\ *Corresponding Author: PAUL T E CUSACK, Independent Researcher, BSc E, DULE, 1641 Sandy \\ Point Rd, Saint John, NB, Canada E2K 5E8, Canada
}

\begin{abstract}
This paper explains how the Riemann Hypothesis is a critical line which results from the Golden Mean Parabola skewed at 60 degrees. The equation gives the roots to the serial of Prime Numbers. The 6-0 degree failure Plane comes from Soil Physics.
\end{abstract}

\section{INTRODUCTION}

The Riemann zeta function $\zeta(s)$ is a function whose argument $s$ may be any complex number other than 1 , and whose values are also complex. It has zeros at the negative even integers; that is, $\zeta(s)=0$ when $s$ is one of $-2,-4,-6, \ldots$. These are called its trivial zeros. However, the negative even integers are not the only values for which the zeta function is zero. The other ones are called non-trivial zeros. The Riemann hypothesis is concerned with the locations of these non-trivial zeros, and states that:

The real part of every non-trivial zero of the Riemann zeta function is $1 / 2$.

Thus, if the hypothesis is correct, all the non-trivial zeros lie on the critical line consisting of the complex numbers $1 / 2+i t$, where $t$ is areal number and $i$ is the imaginary unit.

\section{WIKIPEDIA}

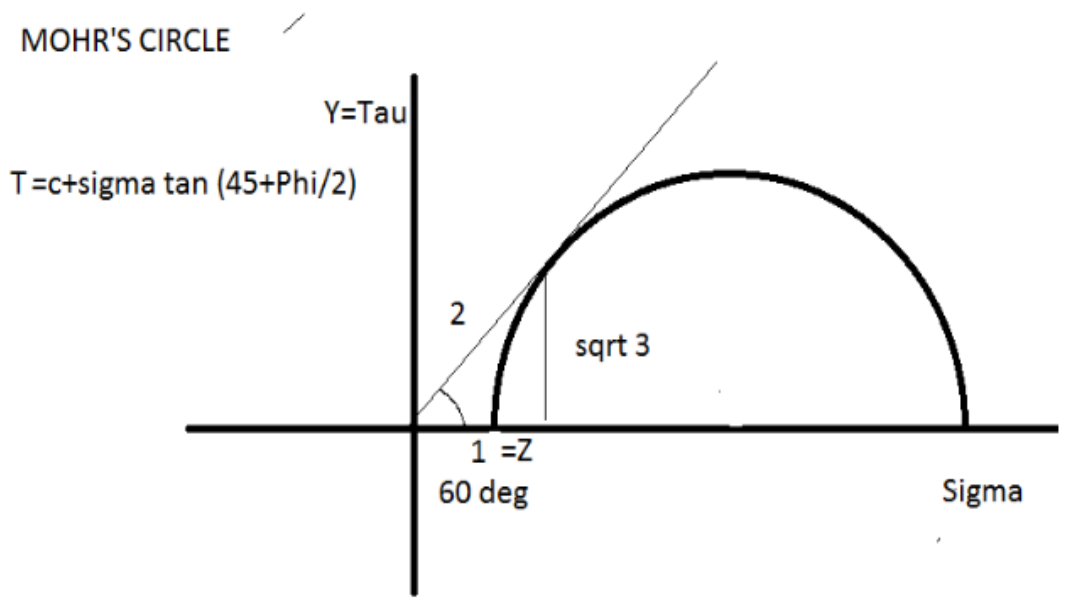

ILLUSTRATION 1 MOHR'S CIRCLE

\section{SOIL MECHANICS}

From Soil Mechanics we know from Mohr-Coulomb Failure:

Tau $=\mathrm{c}+\operatorname{sigma} * \tan (45+\mathrm{Phi} / 2)$

$26.667=0+(\operatorname{sqrt} 3 * \tan (45+30 / 2)$

$=26.667 / \mathrm{sqrt} 3 \mathrm{~h}=0.1539$ 
( $F=26.667$ is the shear failure pressure. Refer to Astrotheology Cusack's Model of the Universe)

$\mathrm{Tau}=\mathrm{F}=\sin$ thet $\mathrm{a}=88.5=\mathrm{k}=$ Permeability of the Universe

So,

$\mathrm{Au} / 2=\sin 60$ degrees

$\left.\mathrm{Z}^{\wedge} 2+(\operatorname{sqrt} 3)^{\wedge} 2\right] / 2=\sin 60$

$\mathrm{Z}=0.5774=1 /$ sqrt $3=\tan 30$ degrees

$\mathrm{Y}=\operatorname{tau}=1 / \tan 30$ degrees $=\cot 30$ degrees $=\operatorname{sqrt} 3=\tan 60$

$\mathrm{y} / \mathrm{z}=$ rise $/$ run $=\mathrm{m}$ in the Y-z PLANE

Tan $60=\mathrm{Y} / \mathrm{z}=3.4641 / 10=\mathrm{e}^{\wedge} \mathrm{z}$

$\mathrm{Y}=\mathrm{e}^{\wedge} \mathrm{Z}$

This is the critical line of the Riemann Hypothesis.

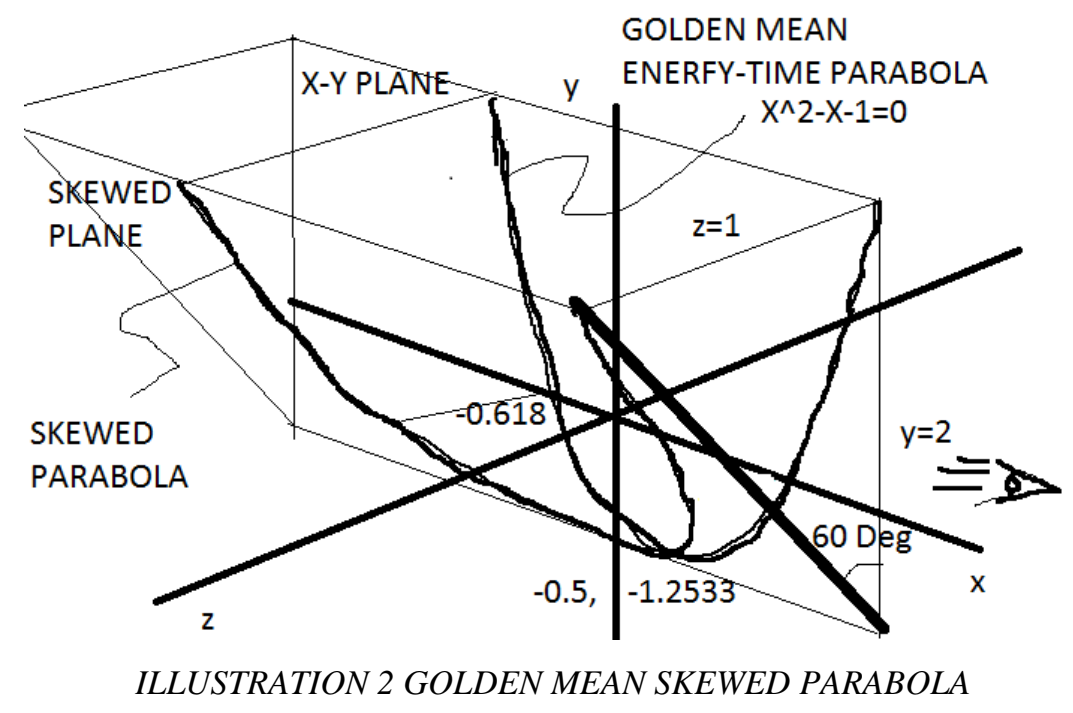

\section{Equation of a Plane}

$a x+b y+c z=0$

\section{EQUATION OF GOLDEN MEAN PARABOLA}

$\mathrm{X}^{\wedge} 2-\mathrm{X}-1=0$

Setting these equal to the skewed plane and the Energy-Time Parabola:

$A x+b y=\sin 60 * z=x^{\wedge} 2-x-1$

There are 5 unknowns; therefore 5 coincident points are necessary. They are:

GOLDEN MEAN ROOTS

$\mathrm{X}=1.618, \mathrm{Y}=0, \mathrm{Z}=0$ (POINT 1)

$\mathrm{X}=-0, .618, \mathrm{Y}=0, \mathrm{Z}=0$ (POINT 2)

MINIMUM POINT OF PARABOLA:

$\mathrm{X}=0.5, \mathrm{Y}=-1.2533, \mathrm{Z}=0$ (POINT 3)

$\mathrm{X}-\mathrm{Y}$ PLANE

$\mathrm{X}=1, \mathrm{Y}=\mathrm{O}, \mathrm{Z}=0$ (POINT 4)

\section{Critical Line}

$\mathrm{Y}=\mathrm{mz}+\mathrm{b}$

$2,0,1$ 
$0=m(1)+b$

$\mathrm{B}=-\mathrm{m}$

Rise over run $=m=2 /-1=-2$

$\mathrm{Y}=2-2 \mathrm{z}$

$\mathrm{X}=2, \mathrm{Y}=0, \mathrm{Z}=1$ (POINT 5)

5. Solve System of 5 EquATIONS; 5 UnKNOWNS

$\mathrm{A}=-1$

$\mathrm{B}=-0.4892 \sim-0.5$

$\mathrm{Z}=$ sqrt 2

$\mathrm{Y}=-0.8154$

$\mathrm{X}=0.9087$

$\mathrm{Y}=2-2 \mathrm{z}$

$\mathrm{A}(1)+\mathrm{b}(0)+\sin 60$ degrees $(0)=1^{\wedge} 2-1-1$

$\mathrm{A}=-1$

$\mathrm{A}(1 / 2)+\mathrm{b}(-1.2533)+\sin 60(0)=(1 / 2)^{\wedge} 2-1 / 2-1$

$\mathrm{B}=0.4892 \sim-1 / 2$

$\mathrm{Ax}+\mathrm{by}+\sin 60 \mathrm{z}=1.618^{\wedge} 2-1.618-1$

$0.8666 \mathrm{z}-0.4892 \mathrm{y}=1.618$

And

$\mathrm{Y}=2-2 \mathrm{z}$

$\mathrm{Y}=2-2$ (sqrt 2)

$\mathrm{Y}=-0.8154$

$0.866 \mathrm{z}(0.4892(2-2 \mathrm{z})=1.618$

$\mathrm{Z}=$ sqrt 2

$\mathrm{Ax}+\mathrm{by}+\sin 60(\mathrm{z})=\mathrm{x}^{\wedge} 2-\mathrm{x}-1$

$-\mathrm{x}+\mathrm{by}+\sin 60 \mathrm{z}=\mathrm{x}^{\wedge} 2-\mathrm{x}-1$

$(-1 / 2)(0.8154)+0.866($ sqrt 2$)=\mathrm{x}^{\wedge} 2$

$\mathrm{X}=0.0987$

6. Critical Line

$\mathrm{m}=-2 / 1=-2$

$\mathrm{Y}=\mathrm{mx}+\mathrm{b}$

$\mathrm{Y}=2-2 \mathrm{Z}$

$\mathrm{Y}=2-2(1 / 2)$

$\mathrm{y}=1$

$Y^{\prime}=-2$

$y-y^{\prime}-2 z$

$y=y^{\prime}-2(1 / 2)$

yy'=-1

$\mathrm{x}=0, \mathrm{y}=1, \mathrm{z}=1 / 2$ 


$$
\begin{aligned}
& E=E^{\prime}-E \\
& E^{\prime}=2 E \\
& E=E^{\prime} / 2 \\
& Y=1 / 2 y^{\prime} \\
& Y=y^{\prime} \\
& Y=e^{\wedge} z \\
& Y=y^{\prime}\left(2 e^{\wedge} z\right) \\
& Y=y^{\prime}+C 1 \\
& Y=e^{\wedge} z \\
& Y=e^{\wedge} z \\
& E^{\wedge} 1=e^{\wedge}(-1)+C 1 \\
& C 1=0.23504
\end{aligned}
$$

Number System based on 10:

Ln C1) $=3.157 \sim \mathrm{Pi}$

$\mathrm{Y}=\mathrm{e}^{\wedge} \mathrm{Z}+\mathrm{Ln}(\mathrm{Pi})$

$\mathbf{Y}=\mathbf{e}^{\wedge} \mathbf{z}+\mathbf{P i}$

7. This is the Critical Line of Prime Numbers

PRIME NUMBERS CALCULATED FROM EQUATION

$\mathrm{Y}=\mathrm{e}^{\wedge} \mathrm{Z}+\mathrm{Pi}$

$\mathrm{Y}^{\prime}=\mathrm{e}^{\wedge} \mathrm{Z}$

$\mathrm{Y}=\mathrm{y}^{\prime}=\mathrm{m}=-2$

$\mathrm{X}^{\wedge} 2-\mathrm{x}-1=0$

$(-2)-2(-2)-1=5$ PRIME

$\mathrm{Z}^{\wedge} 2-\mathrm{z}-1=-3$

$\mathrm{Z}=1,-3$ (Prime)

$\mathrm{Z}^{\wedge} 2-\mathrm{Z}-1=-7$

$\mathrm{Z}=-2,3$

$\mathrm{Z}^{\wedge} 2-\mathrm{z}-1=-11$

$Z=,-4,3$

etc.

THIS, THEN IS THE CRITICAL LINE OF THE SOLUTION TO THE PRIME NUMBERS ACCORDING TO THE REINMANN HYPOTHESIS.

\section{IMAGinaRY NUMber=CONJUGate OF THE GOLDEN MEAN}

Now,

$1 / 2+$ it

$1 / 2+($ sqrt -1$) \mathrm{z}$

GOLDEN MEAN EQUATION:

$\mathrm{X}=1 /[\mathrm{X}-1]$

$1+\mathrm{i}=1 /[1+\mathrm{i}]$ 


$$
\begin{aligned}
& \mathrm{X}=1 /[\mathrm{x}-1] \\
& (1+\mathrm{i})=1 /[1+\mathrm{i})-1 \\
& (1+\mathrm{i})=1 / \mathrm{i} \\
& (1+\mathrm{i}) * \mathrm{i}=1 \\
& \mathrm{I}^{\wedge} 2-\mathrm{i}-1=0
\end{aligned}
$$

Roots (Golden Mean and the Conjugate of the Golden Mean)

$1.618,--0.618$

$\mathrm{I}=\operatorname{sqrt}(-1)=-0.618$

THE UIMAGINARY NUMBER IS EQUAL TO -0.618

$1 / 2+(-0.618)($ sqrt 2$)$

$=0.374=1 / \sin 60$ degrees

$(1 / \mathrm{m})+i^{*} 1 / \sin 60$ degrees $)=1 / 2+i t$

\section{THE UNIVERSE}

The Universe exists where the only real numbers $=s$ are Prime Numbers. Since,

Y=y' for our universe,

And

$\mathrm{y}=\mathrm{y}^{\prime}=\mathrm{e}^{\wedge} \mathrm{x}$

\section{CRITICAL LINE}

$\left(\mathrm{e}^{\wedge} \mathrm{z}+\mathrm{Pi}\right) / \mathrm{e}^{\wedge} \mathrm{z}$

$1+\mathrm{e}^{\wedge} \mathrm{z} / \mathrm{Pi}$

All Physical Quantities should be divided as such.

$1+\mathrm{e}^{\wedge}(4 / 3) / \mathrm{Pi}=6.93$

$1 / 6.93=0.1442$

$1-0.1442=0.855$

$\operatorname{Sin}(-1)(0.855)=58.84$ degrees $=1.027 \mathrm{rads}=\mathrm{t}$

10. CONCLUSION

THE CRITICAL LINDE FOR THE REIMANN HYPOTHESIS IS $\mathrm{y}=\mathrm{e}^{\wedge} \mathrm{z}+\mathrm{Pi}$

\section{REFERENCES}

[1] ELEMENTS OF SOIL MECHANICS, G. N. SMITH.

[2] ASTROTHEOLOGY, CUSACK'S UNIVERSE, BLOG, BY AUTHOR

Citation: PAUL T E CUSACK, (2019). The Reimann Hypothesis Clay Institute Millenium Problem Solution. International Journal of Scientific and Innovative Mathematical Research (IJSIMR), 7(11), pp. 20-24. http://dx.doi.org/ 10.20431/2347 -3142.0711004

Copyright: (c) 2019 Authors, this is an open-access article distributed under the terms of the Creative Commons Attribution License, which permits unrestricted use, distribution, and reproduction in any medium, provided the original author and source are credited. 